\title{
A STUDY OF INTERSTELLAR REDDENING AT HIGH GALACTIC LATITUDES
}

\author{
A. G. DAVIS PHILIP* \\ Dudley Observatory and The State University of New York at Albany
}

\begin{abstract}
A series of finding lists of A stars in regions at high galactic latitudes has been published in the last few years. Strömgren four-color and $\mathrm{H} \beta$ measures have been made of stars in five of the regions, allowing the interstellar absorption in each to be calculated. The two most important regions are the North Galactic Pole and the South Galactic Pole where color excesses of $E_{B_{-} V}=0.00$ and $E_{B-V}=0.02$ were found respectively. An area in front of the Large Magellanic Cloud has a color excess of $E_{B-V}=0.04$.
\end{abstract}

\section{Introduction}

During the past few years a spectral survey has been made of areas at high galactic latitudes, spaced along four principal galactic longitudes $\left(l=0^{\circ}, 76^{\circ}, 180^{\circ} \text {, and } 290^{\circ}\right)^{* *}$ and every $15^{\circ}$ in galactic latitude. When a majority of the regions in the program have been analyzed, it will be possible to study the distribution of early type stars perpendicular to the galactic plane and to determine the tilts of lines of equal star density within a few kiloparsecs of the Sun.

\section{The Observations}

The distribution of areas is shown in Figure 1, where each of the areas under study is

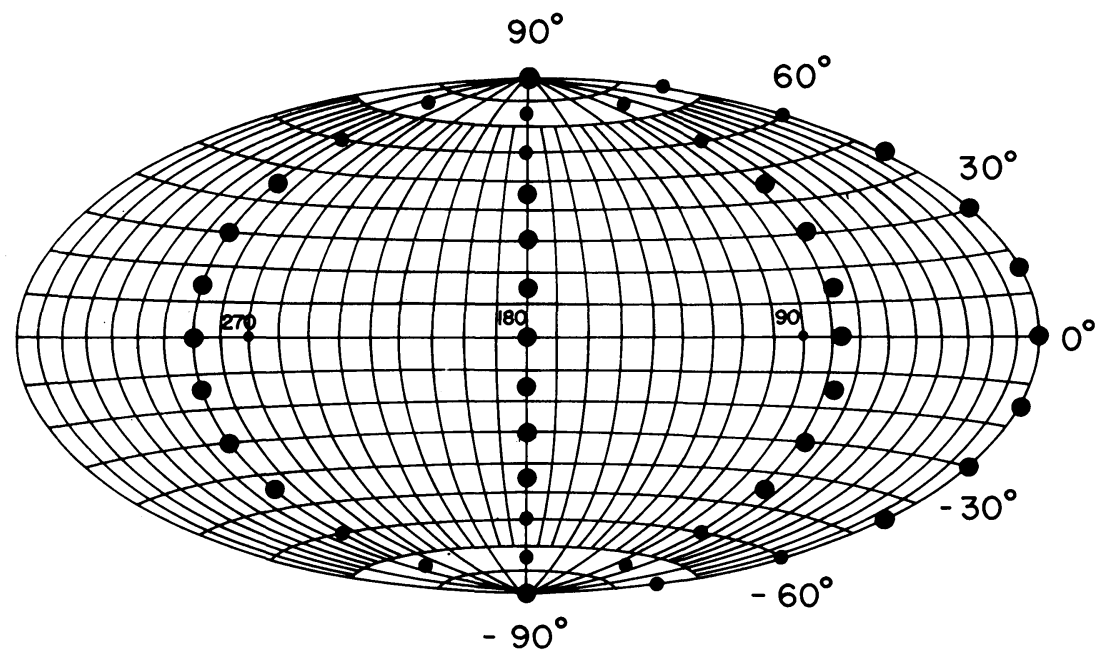

Fig. 1. The areas under investigation in a study of galactic structure perpendicular to the galactic plane plotted by their galactic longitudes and latitudes.

* Visiting astronomer, Kitt Peak National Observatory and Cerro Tololo Inter-American Observatory which are operated by the Association of Universities for Research in Astronomy, Inc.

** New galactic coordinates are used throughout this paper. 
indicated by a solid circle. A series of finding lists of early-type stars, which together constitute a catalogue of stars important in the study of galactic structure, has been published in the Bol. Obs. Tonantzintla y Tacubaya.

Schmidt spectral plates and a set of direct plates covering an area of 25 square degrees, are taken in each area. One hour spectral plates, taken at a dispersion of $280 \AA \mathrm{mm}^{-1}$, on IIaO plates reach approximately $V=13-14$ th mag., depending on the Schmidt telescope used. Plates have been obtained with Schmidt telescopes at the Warner and Swasey Observatory, at the Tonantzintla Observatory, and the Cerro Tololo Inter-American Observatory (Michigan Curtis Schmidt telescope). All the stars with classifiable spectra are recorded and then measured for $B$ or $V$ magnitudes on the direct plates. In order to determine magnitudes from the images on the direct plates, sequences of B and A type stars are set up in each region. The spectral and magnitude data are then combined to yield the stellar density function in each of the directions indicated in Figure 1.

The interstellar reddening must be determined in each region before the stellar density analysis is made. The early-type stars in each area (spectral type A7 or earlier) are measured in the four-color and $\mathrm{H} \beta$ systems. The $y$ magnitude transforms very well to the $V$ magnitude of the $U B V$ system and is used in the determination of the photographic magnitudes. The reddening in each region as a function of distance can be determined by means of a formula derived by Crawford (1970):

$$
(b-y)_{0}=2.943-\beta-0.1\left(\delta m_{1}+\delta c_{1}\right) \text {, }
$$

where $\delta m_{1}=\left(m_{1}\right)_{\mathrm{Hyades}}-\left(m_{1}\right)_{\mathrm{observed}}$, and $\delta c_{1}=\left(c_{1}\right)_{\mathrm{observed}}-\left(c_{1}\right)_{\mathrm{zams}}$. This formula is valid for A stars with $2.70<\beta<2.88$. The color excess, $E_{b-y}=(b-y)_{\text {observed }}-(b-y)_{0}$, can then be plotted as a function of the distance modulus, $m-M$, to determine the run of absorption with distance. Observations of this type have been made in a number of regions in the general program; this paper will summarize the reddenings derived in nine of these.

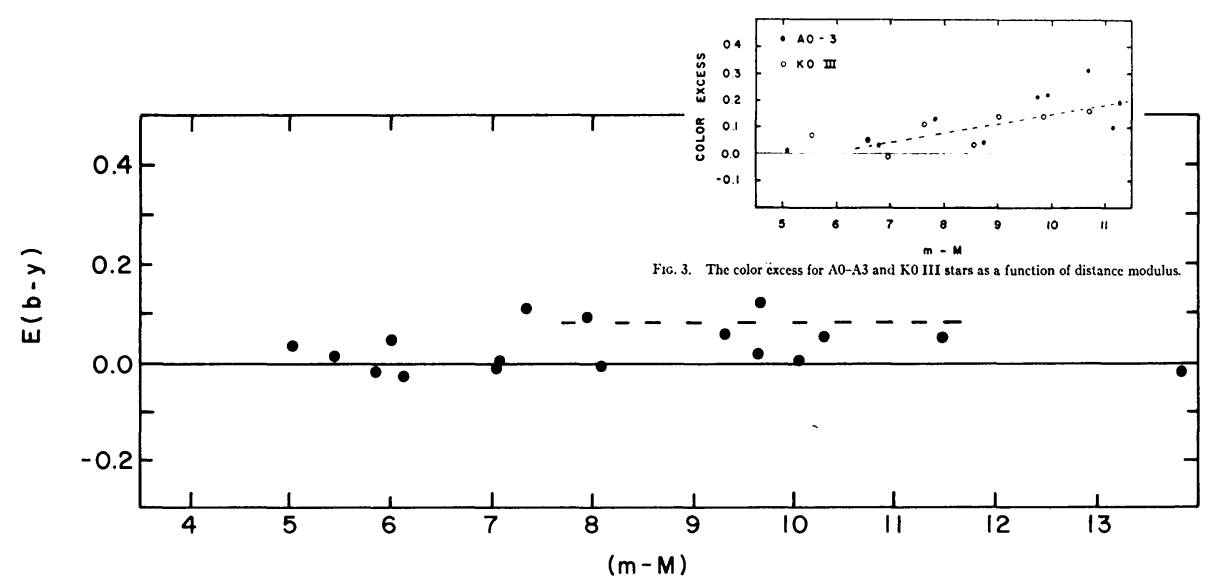

Fig. 2. The color excess in 1HLF2 plotted against the distance modulus. The insert in the upper right is an estimate (Philip, 1966) derived from Schmidt spectral types and UBV photometry. 


\section{The Reddening}

The first area to be studied was 1 HLF2 $\left(l=76^{\circ}, b=-30^{\circ}\right)$ (Philip, 1966). In this study the reddening was derived by using the Schmidt spectral types to yield an intrinsic $B-V$ color, which was then subtracted from the observed $B-V$ color. At a distance modulus of 11 , a color excess of $0.2 \mathrm{mag}$. was derived. More recently these same stars have been measured in the four-color and $\mathrm{H} \beta$ systems and a color excess of $E_{B-V}=0.07$ derived. (The A and B type stars in 1HLF2 are still being measured and the derived reddening estimate may change slightly in the final analysis.) The two estimates of the reddening are shown in Figure 2 in which the color excess, $E_{b-y}$, is plotted against the distance modulus. The small insert in the upper right is a reproduction of Figure 3 from Philip (1966) in which $E_{B-V}$ is plotted vs $m-M . E_{b-y}$ is plotted vs $m-M$ in the large figure. $\left(E_{b-y}=0.7 E_{B-V}\right)$. The observations reported here were made in the fourcolor system; the color excesses have been converted to $E_{B-V}$ since most of the reddenings reported in the literature are in the $U B V$ system.

The reddening in $4 \mathrm{HLF} 6\left(l=180^{\circ}, b=-60^{\circ}\right)$ is presented in Figure 3. In this region $E_{B-V}=0.013$. The reddening at the SGP is presented in Figure $4\left(E_{B-V}=0.002\right)$. The

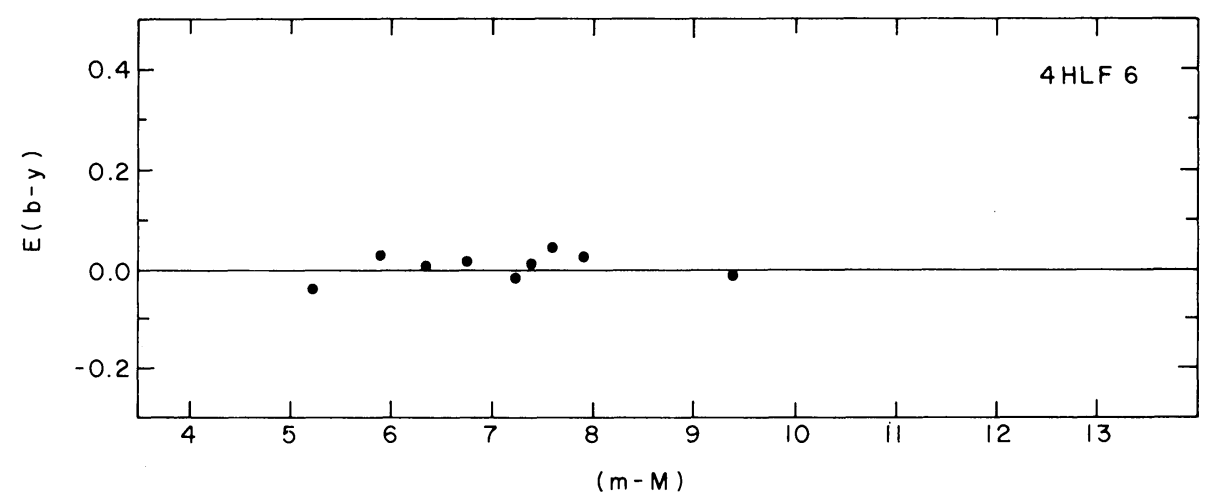

Fig. 3. The color excess in 4HLF6.

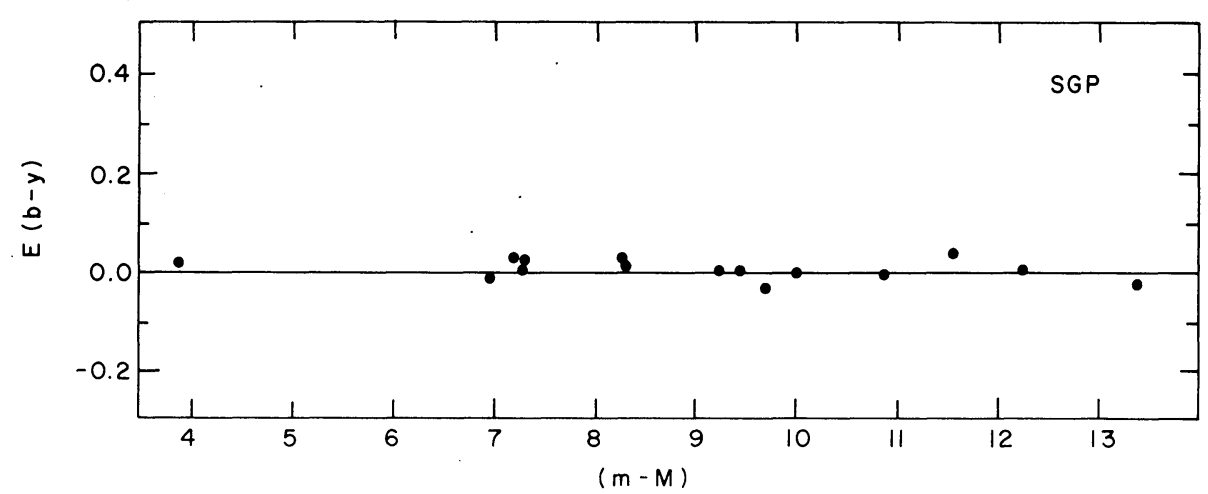

Fig. 4. The color excess at the SGP. 


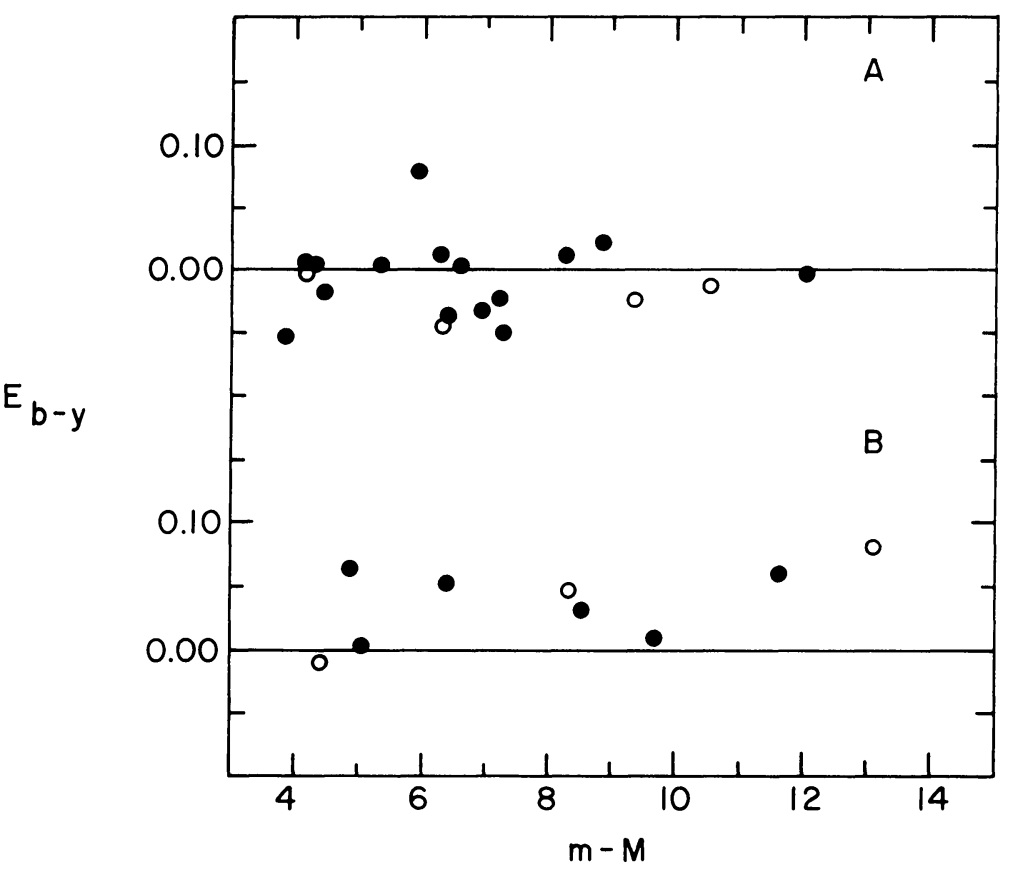

Fig. 5. The color excess at the NGP. The regions A and B are defined in Figure 6.

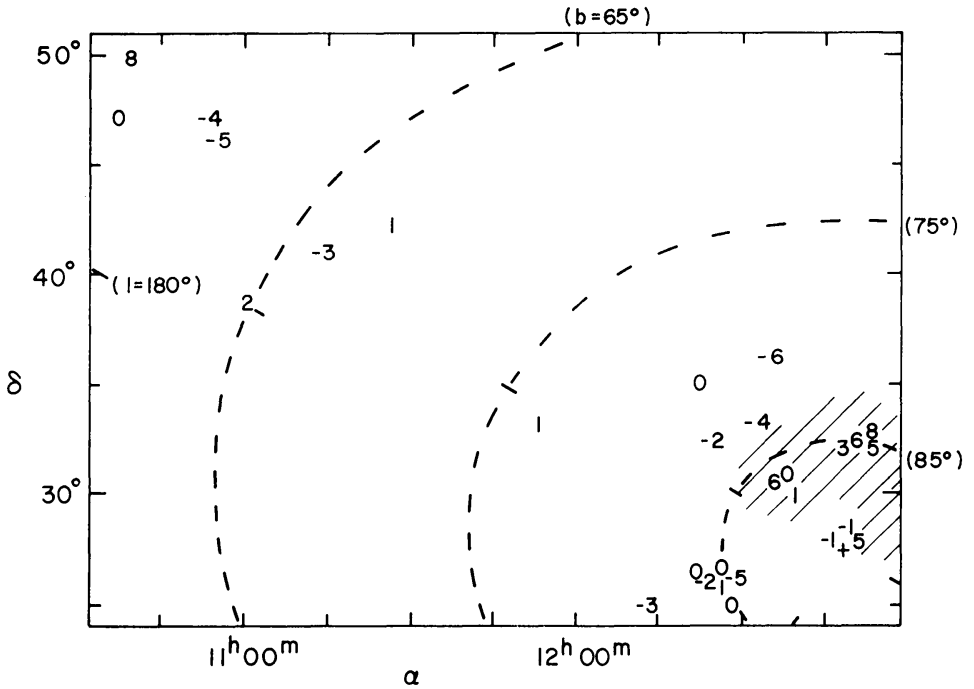

Fig. 6. The distribution of color excesses at the NGP. Area B is a small area just North of the NGP.

reddening at the NGP is presented in Figure 5 and 6, (Figures 1 and 2 of Philip and Tifft, 1971). The color excesses in two regions $A$ and $B$ are shown as a function of distance modulus in Figure 5. In A, $E_{B-V}=0.05$, in B, $E_{B-V}=0.00$. (Region B is indi- 


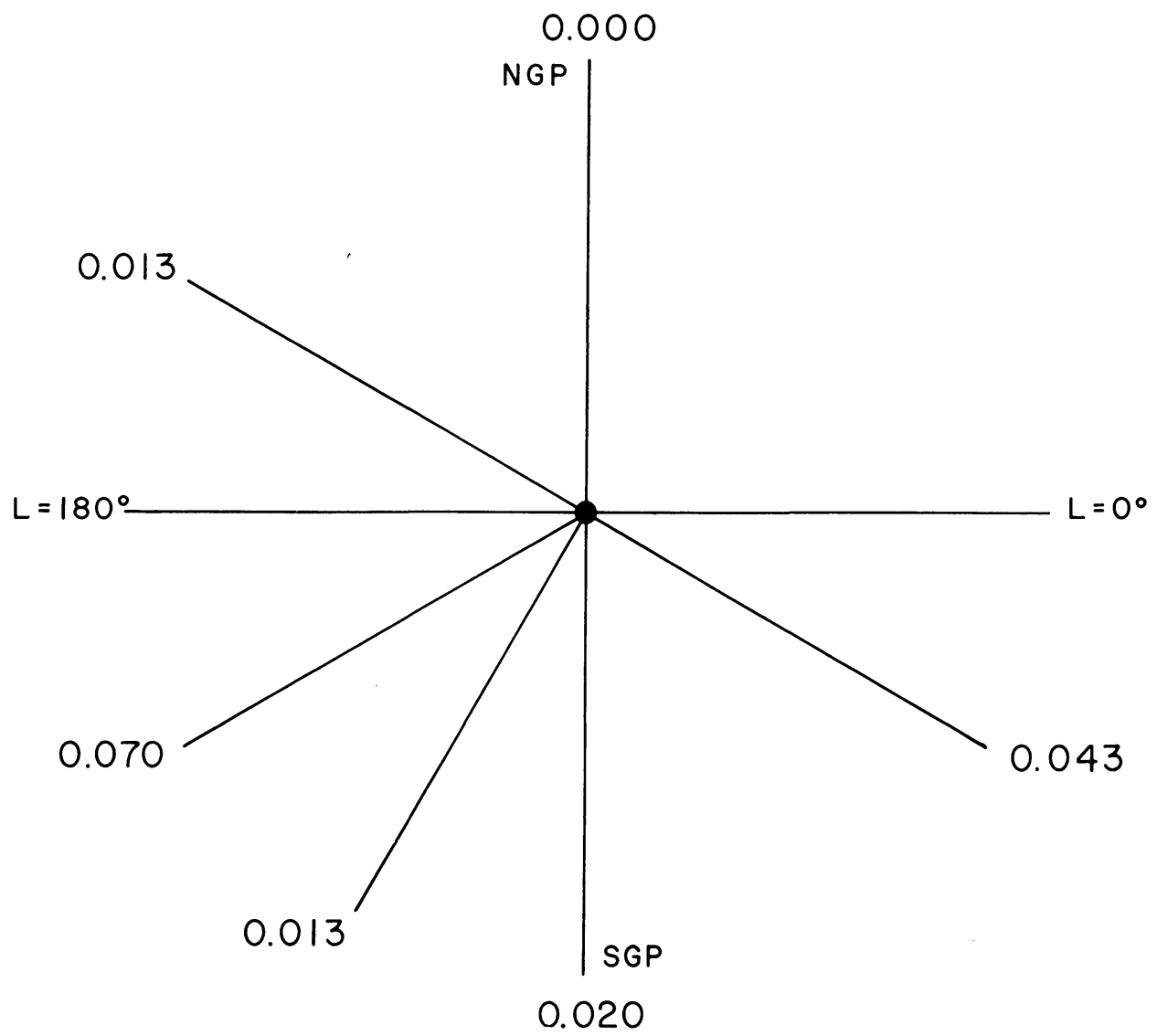

Fig. 7. $E_{\mathrm{B}-\mathrm{v}}$ for certain galactic latitudes at galactic longitudes $0^{\circ}$ and $180^{\circ}$.

cated in Figure 6 as the diagonally shaded area.) The hollow circles in Figure 5 indicate stars with $2.88<\beta<2.91$ which are slightly outside the limits set by Crawford (1971). In Figure 6, the NGP is marked by a small cross in the lower right. The numbers indicate the values of $b-y$ to the nearest hundredth.

The marked increase in accuracy of estimates of the color excess, made by means of the $b-y, \beta$ relation as a function of distance modulus relative to estimates of the color excess made from knowledge of the Schmidt spectral type and $B-V$ color, can be seen by comparing the scatter of the points in Figures 2-5 with the scatter in the small insert in Figure 2. The major cause of the increased scatter is due to the fact that the grid of possible spectral types is not fine enough at the low dispersion of Schmidt spectral types. The difference also may be due partly to small systematic errors in the Schmidt spectral types, both as a function of magnitude and temperature class (in the range $\mathrm{A} 0-\mathrm{A} 7)$ which are now under study.

The estimates of $E_{B-V}$ in nine regions are summarized in Figures 7 and 8 . Areas at $l=0^{\circ}$ and $b=-30^{\circ}, \pm 90^{\circ}, l=180^{\circ}$ and $b= \pm 30^{\circ}$ and $-60^{\circ}$, are summarized in Figure 


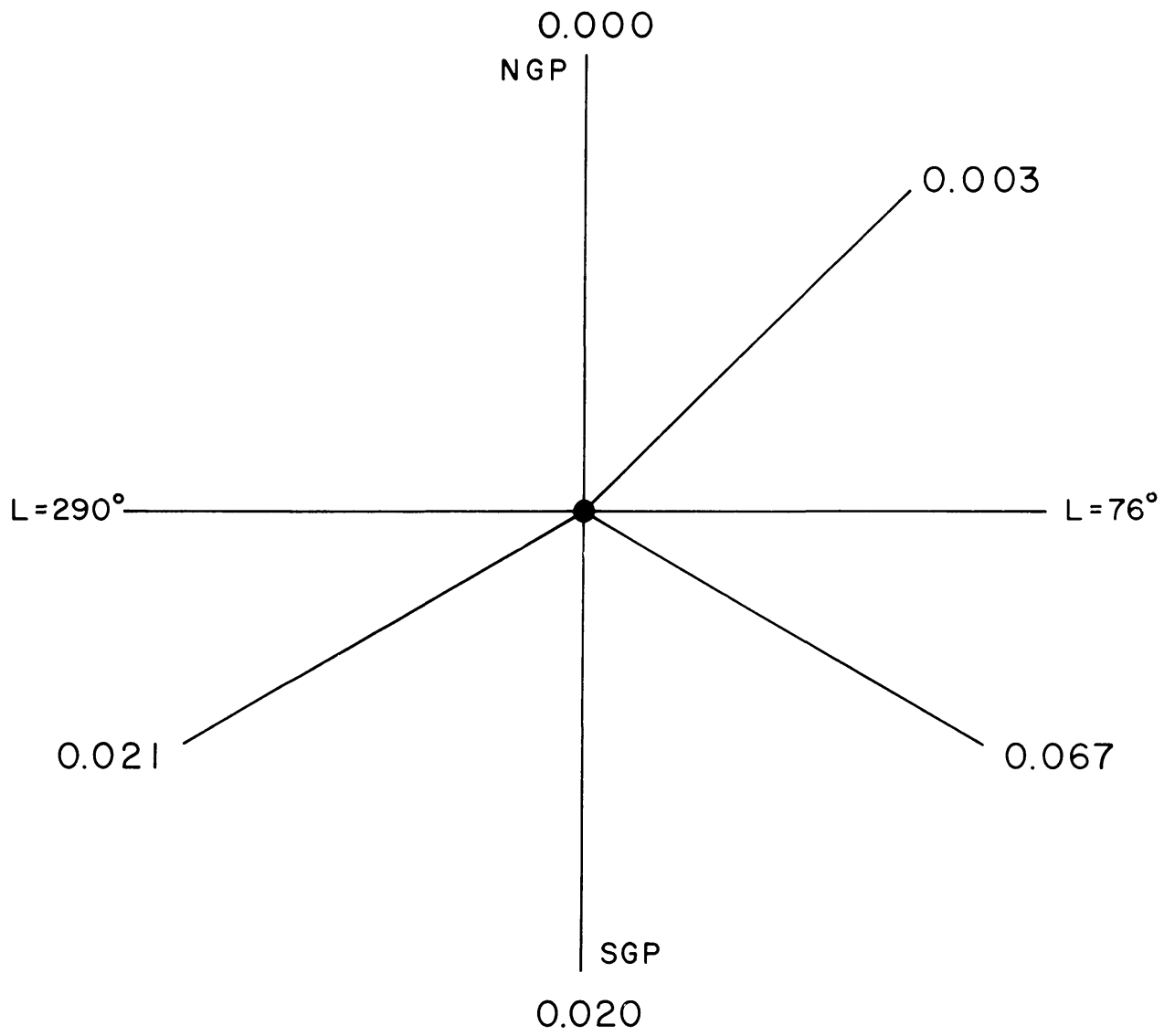

Fig. 8. $E_{\mathrm{B}-\mathrm{v}}$ for galactic latitudes at galactic longitudes $76^{\circ}$ and $290^{\circ}$.

7. The number at each position is the estimate of $E_{B-V}$ in that region. Areas at $l=76^{\circ}$ and $b=+45^{\circ}$, and $-30^{\circ}$, and $l=290^{\circ}, b=-30^{\circ}$ are shown in Figure 8 .

There is a smooth progression from very low reddenings at the galactic poles to estimates of a few hundredths at latitudes of $\pm 30^{\circ}$. As more finding lists of early-type stars are completed and the stars in them measured in the four-color and $\mathrm{H} \beta$ systems, the reddening at high galactic latitudes can be mapped more exactly. At the present time, it can be seen that the reddening at the galactic poles is very small which means that measurements of colors of stars in these areas are very nearly the intrinsic colors. In agreement with current ideas that the Sun is a few tens of parsecs north of the galactic plane, the data suggest that absorption north of the plane is less than that to the south.

\section{Acknowledgements}

The directors of Kitt Peak National Observatory, Cerro Tololo Inter-American Observatory, and the Tonantzintla Observatory are thanked for their generous 
allotment of telescope time for this project. The project was partially supported by the National Science Foundation and the SUNYA Research Foundation.

\section{Appendix}

A summary of the finding lists of early-type stars published and in preparation will be found below

$\begin{array}{lllcccc}\text { Paper } & \text { Reference } & \text { Area } & l & b & \begin{array}{l}\text { No. of } \\ \text { stars }\end{array} & \begin{array}{l}\text { Area of survey } \\ \text { sq deg }\end{array} \\ \text { I } & \text { Philip (1967) } & \text { 1HLF4 } & 76^{\circ} & -45^{\circ} & 33 & 25 \\ \text { II } & \begin{array}{l}\text { Philip and Sanduleak } \\ \text { (1968) }\end{array} & \text { SGP } & -90^{\circ} & & 180 & 230 \\ \text { III } & \text { Philip and Drilling (1970) } & \text { 4HLF4 } & 180^{\circ} & -45^{\circ} & 88 & 45 \\ \text { IV } & \text { Drilling and Philip (1970) } & \text { 3HLF4 } & 0^{\circ} & -45^{\circ} & 146 & 45 \\ \text { V } & \text { Philip and Relyea (1971) } & \text { 1HLF3 } & 76^{\circ} & +45^{\circ} & 16 & 19 \\ \text { VI } & \text { Philip and Stock (1972) } & \text { Extended } & 22^{\circ} & -45^{\circ} & & \\ & & \text { SGP } & 225^{\circ} & -50^{\circ} & 539 & 434 \\ & & & & & 1002 & 798\end{array}$

\section{References}

Crawford, D. L.: 1970, in A. Slettebak (ed.), Stellar Rotation, D. Reidel, Dordrecht, Holland, p. 144. Drilling, J. S. and Philip, A. G. D.: 1970, Bol. Obs. Tonantzintla Tacubaya 4, 307.

Philip, A. G. D.: 1966, Astrophys. J. Suppl. 12, 391.

Philip, A. G. D.: 1967, Bol. Obs. Tonantzintla y Tacubayay 4, 215.

Philip, A. G. D. and Sanduleak, N.: 1968, Bol. Obs. Tonantzintla y Tacubayay 4, 253.

Philip, A. G. D. and Drilling, J. A.: 1970, Bol. Obs. Tonantzintlay y Tacubaya 4, 297.

Philip, A. G. D. and Relyea, L. J.: 1971, Bol. Obs. Tonantzintla y Tacubayay 5, 69.

Philip, A. G. D. and Tifft, L. E.: 1971, Astron. J. 76, 567.

Philip, A. G. D. and Stock, J.: 1972, Bol. Obs. Tonantzintla y Tacubaya 6, 201. 